\title{
Efecto de la lateralización de la embarazada en el gasto cardiaco medido por ecografía transtorácica
}

\author{
JUAN IGNACIO DE BRAHI', FRANCO ARESU', DOMINGO BALDERRAMO', SILVINA LONGO'
}

\begin{abstract}
Effect of lateralization of the pregnant women in the cardiac output by transthoracic echocardiography

Background: Aorta-caval compression in supine position was recognized as a cause of hypotension in the last trimester of pregnancy in 1953. This compression can cause hemodynamic changes and utero-placental hypoperfusion, so it is recommended to perform the lateral position in the term pregnant woman. Materials and Methods: We studied 60 patients who were scheduled for elective cesarean section. Prior to the anesthetic induction, in dorsal decubitus (DD), the left ventricular outflow tract (LVOT) was measured in the parasternal long axis, and then the velocity-time integral (VTI) was obteined from the Apical 5-chamber view. After that the patients were placed in the left lateral decubitus position (LLD) and VTI was re-measured after 3 minutes. The estimation of the Cardiac output(CO) was made by the formula: VTI x (r2 LVOT x 3.14) x HR. Results: The average age was 33.7 years. The $C O$ was higher when the patient was in LLD compared to DD $(6,727 \pm 234 \mathrm{ml} \mathrm{ml} v$ s $5,795 \pm 276 \mathrm{ml}, \mathrm{p}<0.001)$. This increase was $16.2 \%$. In the LLD position. Discussion: This study evidenced that echocardiography is a good tool for estimating $\mathrm{CO}$ during the lateralization of the pregnant woman. Conclusion: In patients with full-term pregnancy, echocardiography showed a significant increase in CO in LLD compared to DD, which is why we affirm the veracity of this maneuver.
\end{abstract}

\section{Key words:}

Echocardiography, pregnancy, aorto-caval compression, cardiac output.

Servicio de Anestesiología, Hospital Privado Universitario de Córdoba, Argentina.

Av. Naciones Unidas 346, Córdoba, Argentina.

Los autores no declaran conflictos de interés

El trabajo no contó con fuentes de financiamiento externo

Fecha de recepción: 10 de enero de 2018

Fecha de aceptación: 19 de febrero de 2018

ORCID

https://orcid.org/0000-0002-0491-2651

\section{Correspondencia:}

Juan Ignacio De Brahi

25 de Mayo 1076 7D, Córdoba, Argentina; 0540351152370117

Email: juanidebrahi@hotmail.com 


\section{RESUMEN}

Introducción: La compresión aórtica-cava en posición supina fue reconocida como causa de hipotensión en el último trimestre del embarazo en 1953. Esta compresión puede causar cambios hemodinámicos e hipoperfusión útero-placentaria, por lo que se recomienda la posición lateral en el término embarazada mujer. Material y Métodos: Estudiamos a 60 pacientes que se programaron para cesárea electiva. Antes de la inducción anestésica, en el decúbito dorsal (DD), se midió el tracto de salida del ventrículo izquierdo (TSVI) en el eje largo paraesternal, y luego se obtuvo la integral de velocidad-tiempo (IVT) desde la vista Apical de 5 cámaras. Después de eso, los pacientes se colocaron en la posición de decúbito lateral izquierdo (LLD) y se volvió a medir la IVT después de 3 minutos. La estimación del gasto cardíaco (GC) se realizó mediante la fórmula: IVT x ( $r 2$ LVOT x 3.14) x FC. Resultados: La edad promedio fue 33,7 años. El GC fue más alto cuando el paciente estaba en LLD en comparación con DD (6.727 $\pm 234 \mathrm{ml} \mathrm{ml}$ frente a $5.795 \pm 276 \mathrm{ml}, \mathrm{p}<0,001)$. Este aumento fue $16,2 \%$ en la posición LLD. Discusión: Este estudio evidenció que la ecocardiografía es una buena herramienta para estimar el CG durante la lateralización de la mujer embarazada. Conclusión: En pacientes con embarazo a término, la ecocardiografía mostró un aumento significativo de CO en LLD en comparación con DD, por lo que afirmamos la veracidad de esta maniobra.

\section{Palabras clave:}

Ecocardiografía, embarazo, compresión aorto-cava, gasto cardíaco.

\section{Introducción}

a compresión aorta-cava en posición supina fue reconocida como causa de hipotensión en el último trimestre del embarazo desde el reporte de Howard et,al en 1953[1].

Dicha compresión puede causar cambios hemodinámicos e hipoperfusión utero-placentaria por lo que se recomienda realizar la inclinación pélvica en la embarazada a término para evitar la compresión aorto-cava[2],[3].Esta es una medida universalmente adoptada, particularmente durante la cesárea[4]. El fenómeno del "shock postural" en posición supina fue descripto en mujeres sanas al final del embarazo desde hace más de 70 años[5].

La American Heart Association recomienda posicionar a las pacientes embarazadas en decúbito lateral izquierdo en un ángulo de 27 a 30 grados usando una "cuña" firme para sostener la pelvis y el tórax (como la cuña de Cardiff por ejemplo)[6], si la lateralización manual del útero es insatisfactoria (Class II b, Level of Evidence C)[7].

Cabe destacar que una gama de modalidades, tanto invasivas como no invasivas, se han utilizado para cuantificar los efectos hemodinámicos globales de la posición supina al final del embarazo; los es- tudios anteriores de la década de 1960 se basaron en más técnicas invasivas de termodilución. Los cambios típicos asociados con la posición supina incluyen disminución de la presión auricular derecha, aumento de la resistencia vascular sistémica, aumento de la frecuencia cardiaca (FC) y disminución del Índice Cardiaco (IC) del 5\% al 21\%, en comparación con la posición lateral o semilateral. La Presión Arterial parece mantenerse por un aumento concomitante en la resistencia vascular periférica.

Sin embargo, ha habido poca uniformidad entre los estudios que describen que el IC materno cambia con la posición durante el final del embarazo. Específicamente, los estudios han variado con respecto a la modalidad utilizada, lateralidad de posicionamiento, y el grado de inclinación. Además, muchas técnicas utilizadas para medir IC no han sido validados durante el embarazo[7].

Un método fidedigno para evaluar esta compresión podría ser la ecocardiografía transtorácica, estimando las modificaciones del gasto cardiaco (GC) y así comprobar de manera no invasiva si la lateralización en pacientes embarazadas cambia el GC. El objetivo principal fue entonces, estimar con ecocardiografía transtorácica si existen cambios en GC en DD (decúbito dorsal) y en DLI (decúbito lateral izquierdo). 


\section{Materiales y Métodos}

El siguiente protocolo fue aprobado por el Comité de Ética y el consentimiento informado fue obtenido por escrito por parte del paciente el día previo al procedimiento.

La investigación siguió los lineamientos de la Declaración de Helsinki, Finlandia, de la Asociación Médica Mundial (1964); revisada y enmendada por la 64 Asamblea General de Fortaleza, Brasil 2013.

Se realizó un estudio descriptivo prospectivo entre diciembre de 2015 y julio de 2016. Se estudiaron 60 pacientes de sexo femenino cursando el tercer trimestre de embarazo y que fueron programadas para operación cesárea durante la mañana y con 6 a 8 horas de ayuno. La edad para ser incluídos al protocolo fue a partir de 18 años. El presente estudio se llevo a cabo en la sala de inducción anestésica previo al ingreso a la sala de operaciones, realizando mediciones especificas con ecocardiografía transtorácica. Se excluyeron las cesáreas de urgencia y la falta de progresión del trabajo de parto que culminó en cesárea.

Las mediciones fueron realizadas por el mismo operador anestesiólogo formado en ecocardiografía utilizando un equipo Sonosite Turbo (Fujifilm X-3421).

Previo a la inducción anestésica, en decúbito dorsal (DD), se midió el tracto de salida del ventrículo izquierdo (TSVI) en ventana paraesternal eje largo e integral velocidad-tiempo (IVT) en ventana Apical 5 cámaras. Luego se colocó a las pacientes en decúbito lateral izquierdo (DLI) y después de 3 minutos se volvió a medir IVT. Para medir IVT se utilizó Doppler Pulsado en tracto de salida de ventrículo izquierdo.

La estimación del GC se realizó por la fórmula: IVT $x\left(r^{2}\right.$ TSVI $\left.x \pi\right)=$ Volumen eyectivo. Luego Volumen eyectivo $x$ frecuencia cardíaca $(F C)=$ Gasto Cardíaco.

Un investigador que no participó de las mediciones ecocardiográficas registró todos los datos hemodinámicos, estos fueron la frecuencia cardíaca en DS y en DD y presión arterial sistólica y diastólica en DS y DD. Ninguna paciente manifestó molestias en ambas posiciones, se preguntó expresamente por náuseas, vómitos, palpitaciones y/o mareos.

Video 1. Estimación del IVT a nivel del tracto de salida de la válvula aórtica se utiliza Doppler para medir la velocidad y el equipo calcula mediante un software la integral de velocidad en tiempo.

Para el análisis estadístico se utilizo el software SPSS (versión 20.0). El tamaño muestral se calculó en base a una diferencia entre el GC basal versus el GC

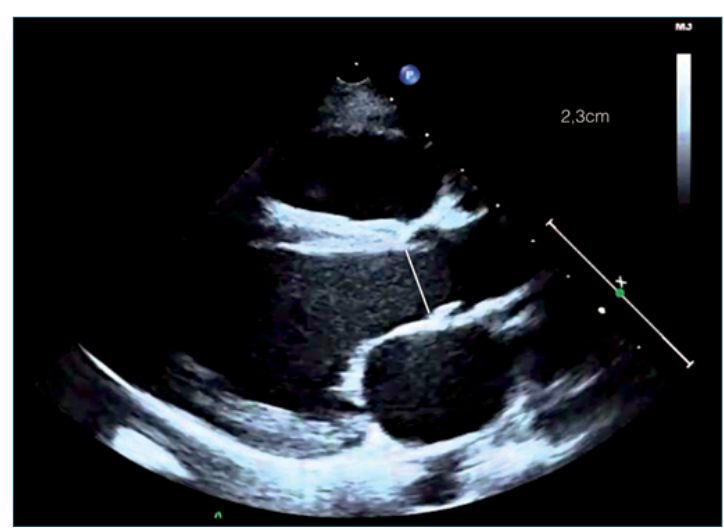

Figura 1. Ventana paraesternal eje largo: Medición del diametro del Tracto de salida del VI.

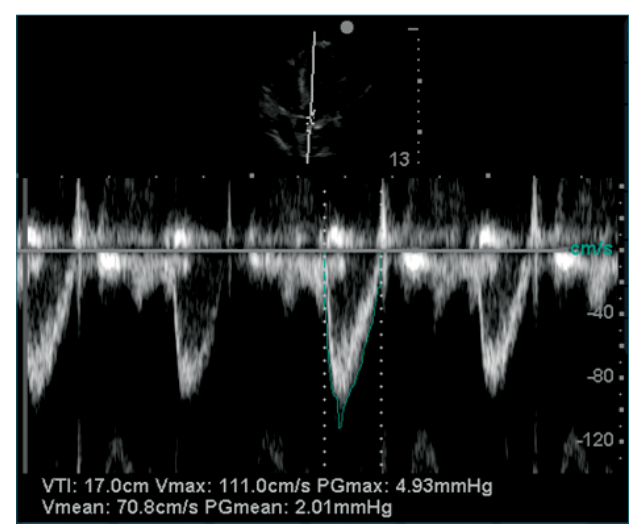

Figura 2. Ventana apical 5 cámaras: IVT en tracto de salida del VI con Doler Pulsado, que en este caso fue de $17 \mathrm{~cm} / \mathrm{seg}$.

en DLI de un 10\%, basándose en la experiencia clínica del grupo de anestesiólogos dedicados a obstetricia y este fue de 25 pacientes por grupo. Se utilizó t Test para muestras pareadas Se consideró como estadísticamente significativo un $p<0,05$.

\section{Resultados}

Se incluyeron 60 pacientes. Se excluyeron 5 pacientes por no poder obtenerse una adecuada visualización de la ventana apical 5 cámaras. La edad media fue 33,7 \pm 11 (rango 21 a 38) años.

Con respecto al Gasto Cardiaco, este fue superior cuando se evaluó en DLI comparado a DD (6.727 \pm $234 \mathrm{ml}$ vs $5.795 \pm 276 \mathrm{ml} ; \mathrm{p}<0,001)$. Dicho aumento fue del $16,2 \%$ en DLI respecto al basal.

Por otro lado, se observó un aumento pequeño 
pero significativo de la presión arterial media en pacientes en DLI comparado con DD $(90 \pm 11 \mathrm{mmHg}$ vs $88 \pm \mathrm{mmHg}$ ). Dicho aumento represento un cambio del $1,14 \%$, sin alcanzar significancia estadística.

\section{Discusión}

Este estudio evidenció que se puede estimar el GC en la paciente embarazada utilizando ecocardiogrfía transtorácica. También se demostró que la lateralización de la paciente embarazada es una buena medida para aumentar el GC.

Higuchi et al[8], realizaron un estudio en el cual observaron a través de resonancia magnética la compresión aorto-cava en mujeres a término, colocándolas en posiciones supina e inclinadas lateralmente y concluyeron que $15^{\circ}$ de inclinación hacia la izquierda es suficiente para aliviar la compresión de la vena cava inferior. Así mismo se observó que no existe una franca compresión aórtica, por el útero grávido, aunque la aorta distal usualmente está mal visualizada. En general, el grado y la importancia de la compresión aórtica por el útero grávido durante anestesia neuroaxial, en caso de hipotensión, y durante las contracciones uterinas, también sigue siendo discutible[9].

Otro estudio demostró que en pacientes bajo anestesia espinal no se encontraron diferencias en el estado ácido-base neonatal entre las mujeres aleatorizadas para estar en posición decúbito lateral izquierdo en $15^{\circ}$; o para estar en posición supina, si la presión arterial sistólica materna se mantiene al inicio con un cristaloide y una infusión profiláctica de fenilefrina[10]. Esta revisión presentó una nueva mirada a las décadas de evidencia que rodean este tema y propuso una reevaluación de las pautas actuales con respecto a las prácticas arraigadas. Un estudio novedoso fue el de Lee et al[5], que utilizando Doppler suprasternal en mujeres con edad gestacional media 38,4 semanas $(n=157)$, compararon 4 niveles de inclinación lateral izquierda $\left(0^{\circ}\right.$ [supina], $7,5^{\circ}, 15^{\circ}$ y $\left.90^{\circ}\right)$. El IC materno fue en promedio un $5 \%$ más alto en las posiciones $\geq 15^{\circ}(\mathrm{P}=.001)$ en comparación con la posición supina; y cabe destacar que 11 de 157 (7\%) pacientes mostraron disminución $>20 \%$ en $7,5^{\circ}$ y $0^{\circ}$ de inclinación en comparación con los $15^{\circ}$ posición inclinada izquierda, lo que fue considerado por los autores como una compresión severa.

Por ultimo, queremos mencionar que en un grupo de 5 pacientes realizamos la medición de la IVT con Doppler pulsado a nivel de la ventana supraesternal, en DD y DLI respectivamente (Figuras 3 y 4 ). Como se puede ver en las imágenes, el Gasto Cardíaco aumen-

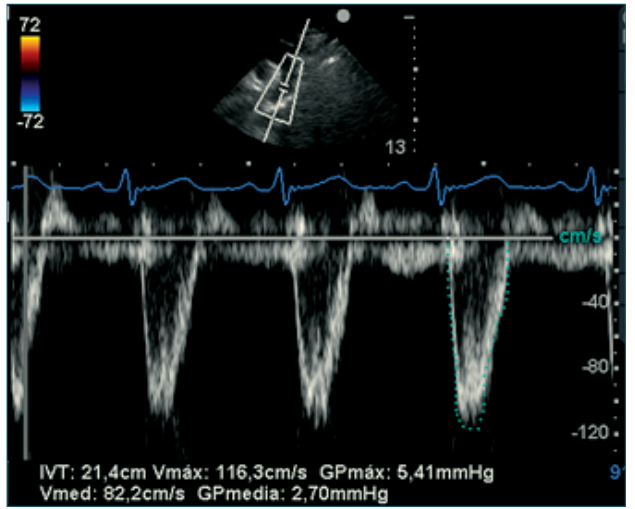

Figura 3. Paciente en DD. Imagen supraesternal. Con Doppler color y pulsado desde el hueco supraesternal flujo de aorta descente: VTI $\times\left(\mathrm{r}^{2}\right.$ TSVI $\left.\times \varpi\right)=$ VOLUMEN EXPULSIVO. IVT $(21,4) \times\left(3,14 \times 1^{2}\right)=67 \mathrm{ml}$. Vol EXPULSIVO $(67) \times$ FC (62) $=\mathrm{GC}=4,1 \mathrm{lits} / \mathrm{min}$.

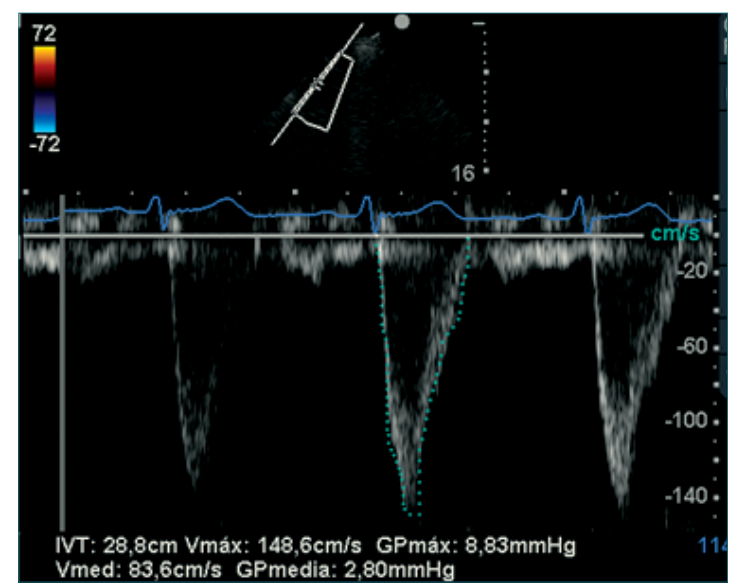

Figura 4. Paciente en DLI. Imagen supraesternal, con doppler pulsado flujo de aorta descentente: IVT $x\left(r^{2}\right.$ TSVI $x " \varpi(3,14) ")=$ VOLUMEN EXPULSIVO. IVT $(28,8) \times(3,14 x$ $1^{2)}=90 \mathrm{ml}$. Vol. expulsivo $(90) \times F C(75)=G C=6,7$ lits $/ \mathrm{min}$.

tó de 4,1 Its en DD a 6,7 Its en DLI. Si bien se necesitan más estudios para evaluar si la variabilidad del IVT, puede ser una medida útil para inferir cambios en el gasto cardíaco. Esta medición es más simple y rápida de realizar que obtener 5 cámaras apical y la vista paraesternal sobre todo en pacientes con abdomen globuloso por el útero grávido.

Las principales limitaciones de este trabajo son la falta de aleatorización en la posición para hacer las mediciones. Con el protocolo planteado, no se puede saber si los valores obtenidos son diferentes si primero se miden con la paciente en DLI vs DD o al revés. 
Otra limitación es que el estudio se hizo en pacientes sin anestesia. Los resultados podrían variar en la paciente con anestesia espinal, que es el momento más crítico[9], que más nos preocupa a los anestesiólogos y que es la motivación de la realización del presente estudio. En parte por esto se comenta que al utilizar vasopresores alfa-1 el efecto de la compresión aortocava pudiera no existir, lo que es correcto. Finalmente, se debe reconocer que el cálculo (estimación) de GC es un resultado subrogante de perfusión útero- placentaria, que es lo más importante a preservar y a evitar que se comprometa con la posición adoptada por la paciente ${ }^{10}$. Según estos resultados, planificamos nuestra próxima investigación para evaluar el GC en la embarazada antes y después del bloqueo regional.

En conclusión las pacientes con embarazo a término la ecocardiografía fue útil para estimar el GC. Se evidenció un aumento del GC en DLI comparado al DD por lo que esta maniobra es realmente de utilidad.

\section{Referencias}

1. Howard BK, Goodson JH, Mengert WF. Supine hypotensive syndrome in late pregnancy. Obstet Gynecol. 1953;1:371-7.

2. Scott DB. Inferior vena caval occlusion in late pregnancy and its importance in anaesthesia. $\mathrm{Br} J$ Anaesth. 1968;40:120-8.

3. Eckstein KL, Marx GF. Aortocaval compression and uterine displacement. Anesthesiology. 1974;40:92-6.

4. Secher NJ, Arnsbo P, Andersen LH, Thomsen A. Measurements of cardiac stroke volume in various body positions in pregnancy and during Caesarean section: A comparison between thermodilution and impedance cardiog- raphy. Scand J Clin Lab Invest. 1979;39:569-76.

5. Lee SW, Khaw KS, Ngan Kee WD, Leung TY, Critchley LA. Haemodynamic effects from aortocaval compression at different angles of lateral tilt in non-labouring term pregnant women. $\mathrm{Br} J$ Anaesth. 2012;109:950-6.

6. Rees GA, Willis BA. Resuscitation in late pregnancy. Anaesthesia. 1988:43:347-9.

7. Rees SG, Thurlow JA, Gardner IC, Scrutton MJ, Kinsella SM. Maternal cardiovascular consequences of positioning after spinal anaesthesia for Caesarean section: Left 15 degree table tilt vs left lateral. Anaesthesia. 2002;57:15-20.
8. Higuchi H., Shunichi T, Kan Z, Ikue F, Makoto O. Effect of Lateral Tilt Angle on the Volume of the Abdominal Aorta and Inferior Vena Cava in Pregnant and Nonpregnant Women Determined by Magnetic Resonance Imaging; Anesthesiology, Volume 122(2), February 2015,p 286-293.

9. Mendonca C, Griffiths J, Ateleanu B, Collis RE. Hypotension following combined spinal-epidural anaesthesia for Caesarean section. Left lateral position vs. tilted supine position. Anaesthesia. 2003;58:428-31.

10. Lee AJ, Landau R. Aortocaval compression syndrome: Time to revisit certain dogmas. Anesth Analg 2017: 125: 1975-85. 